UnIVERSità DI PISA

Dipartimento Di InFormaticA

TeChnical Report: TR-08-08

\title{
Toward a Formal Semantics for Autonomic Components
}

\author{
Marco Aldinucci Emilio Tuosto
}

April 22, 2008

ADDREsS: Largo B. Pontecorvo 3, 56127 Pisa, Italy. TEL: +39 0502212700 FAX: +39 0502212726 



\title{
Toward a Formal Semantics for Autonomic Components
}

\author{
Marco Aldinucci $\quad$ Emilio Tuosto*
}

April 22, 2008

\begin{abstract}
Autonomic management can improve the QoS provided by parallel/ distributed applications. Within the CoreGRID Component Model, the autonomic management is tailored to the automatic - monitoring-driven - alteration of the component assembly and, therefore, is defined as the effect of (distributed) management code.

This work yields a semantics based on hypergraph rewriting suitable to model the dynamic evolution and non-functional aspects of Service Oriented Architectures and component-based autonomic applications. In this regard, our main goal is to provide a formal description of adaptation operations that are typically only informally specified. We contend that our approach makes easier to raise the level of abstraction of management code in autonomic and adaptive applications.
\end{abstract}

\section{Introduction}

Developers of grid applications cannot rely neither on fixed target platforms nor on stability of their status [9]. This makes dynamic adaptivity of applications an essential feature in order to achieve user-defined levels of Quality of Service (QoS). In this regard, component technology has gained increased impetus in the grid community for its ability to provide a clear separation of concerns between application logic and QoS-driven adaptation, which can also be achieved autonomically. As an example, GCM (the Grid Component Model defined within the CoreGRID NoE) is a hierarchical component model explicitly designed to support component-based autonomic applications in highly dynamic and heterogeneous distributed platforms [4].

An assembly of components may be naturally modeled as a graph and, if components are autonomic, the graph can vary along with the program execution and may change according to input data and/or grid hardware status. These changes can be encoded as reaction rules within the component Autonomic Manager (hereafter denoted as $A M$ ). A proper encoding of these rules

\footnotetext{
* Department of Computer Science, University of Leicester, UK
} 
effectively realises the management policy, which can be specific of a given assembly or pre-defined for parametric assemblies (such as behavioural skeletons) $[1,2]$. In any case, the management plan relies on the reconfiguration operation exposed by the component model run-time support.

A major weakness of current component models (including GCM) is that the semantics of these operations are informally specified, thus making hard to reason about QoS-related management of components. In this work

- We discuss few primitives useful for component adaptation; the chosen operations are able to capture typical adaptation patterns in parallel/ distributed application on top of the grid. These are presented as nonfunctional interfaces of components that trigger component assembly adaptation.

- We detail a semantics for these operations based on hypergraph rewriting suitable for the description of component concurrent semantics and assembly evolution along adaptations.

The key idea of our semantical model consists in modeling component-based applications by means of hypergraphs which generalise usual graphs be allowing hyperedges, namely arcs that can connect more than two nodes. Intuitively, hyperedges represent components able to interact through ports represented by nodes of hypergraphs. The Synchronized Hyperedge Replacement (SHR) model specifies how hypergraphs are rewritten according to a set of productions. Basically, rewritings represent adaptation of applications possibly triggered by the underlying grid middleware events (or by the applications themselves).

SHR has been shown suitable for modelling non-functional aspects of service oriented computing $[6,7]$ and is one of the modelling and theoretical tools of the Sensoria project [14]. For simplicity, we consider a simplified version of SHR where node fusions is limited and restriction is not considered. Even if, for the sake of simpleness, the SHR framework used in this work is not the most general available, it is sufficient to give semantics to the management primitives (aka adaptation operations) addressed here. The autonomic manager - by way of these adaptation operations - can structurally reconfigure an application to pursue the (statically or dynamically specified) user intentions in terms of QoS.

\section{Autonomic Components and GCM}

Autonomic systems enables dynamically defined adaptation by allowing adaptations, in the form of code, scripts or rules, to be added, removed or modified at run-time. These systems typically rely on a clear separation of concerns between adaptation and application logic [10]. An autonomic component will typically consist of one or more managed components coupled with a single autonomic manager that controls them. To pursue its goal, the manager may trigger an adaptation of the managed components to react to a run-time change of application QoS requirements or to the platform status. In this regard, an assembly of self-managed components implements, via their managers, a distributed algorithm that manages the entire application. 
The idea of autonomic management of parallel/distributed/grid applications is present in several programming frameworks, although in different flavours: ASSIST [15, 2], AutoMate [13], SAFRAN [5], and GCM [4] all include autonomic management features. The latter two are derived from a common ancestor, i.e. the Fractal hierarchical component model [12]. All the named frameworks, except SAFRAN, are targeted to distributed applications on grids.

GCM builds on the Fractal component model [12] and exhibits three prominent features: hierarchical composition, collective interactions and autonomic management. GCM components have two kinds of interfaces: functional and non-functional ones. The functional interfaces host all those ports concerned with implementation of the functional features of the component. The nonfunctional interfaces host all those ports needed to support the component management activity in the implementation of the non-functional features, i.e. all those features contributing to the efficiency of the component in obtaining the expected (functional) results but not directly involved in result computation. Each GCM component therefore contains an $A M$, interacting with other managers in other components via the component non-functional interfaces. The $A M$ implements the autonomic cycle via a simple program based on reactive rules. These rules are typically specified as a collection of when-event-ifcond-then-adapt_op clauses, where event is raised by the monitoring of component internal or external activity (e.g. the component server interface received a request, and the platform running a component exceeded a threshold load, respectively); cond is an expression over component internal attributes (e.g. component life-cycle status); adapt_op represents an adaptation operation (e.g. create, destroy a component, wire, unwire components, notify events to another component's manager) [5].

We informally describe some common adaptation operations that may be assigned to configuration interfaces are the following:

Migration A component is required to change its running location (e.g. platform, site). The request must include the new location and can be performed while keeping its attached external state (go) or restating from a fresh default state (start).

Replication A component (either composite or primitive) is replicated. Replication operation is particularly targeted to composite components exhibiting the parametric replication of inner components (such as behaviour skeletons), and can be used to change their parallelism degree (and thus their performance and fault-tolerance properties). Replication events are further characterized with respect to their relation with replicated component state, if any. A component replica may be created with a fresh external state, carry a copy of the external state (copy), or share the external state with the source component (share).

Kill A component is killed. Due to this kind of action disconnected components (and in particular storage managers) can subject to garbage collection.

Described primitives make possible the implementation of several adaptation paradigms. In particular, migration may be used to adapt the application to 
changes of grid topology as well as to performance drop of resources. Replication and kill may be used to adapt both data and task parallel computation. In particular, replication with share enables the redistribution of sub-task in data parallel computations; replication with copy enables hot-redundancy. Both stateful and stateless farm computation (parameter-sweeping, embarrassingly parallel) may be reshaped both in parallelism degree and location run by using replication and kill.

Example 2.1 Let P, C, SF, S, AM, $\mathrm{W}_{1}, \mathrm{~W}_{2}, \mathrm{~W}_{3}$ components (Producer, Consumer, Stateful Farm ${ }^{1}$, Storage, Autonomic Manager, and Workers); $\mathrm{L}_{1} \cdots \mathrm{L}_{8}$ locations. Thee kinds of bindings are used in the assembly (see also Sec. 4).

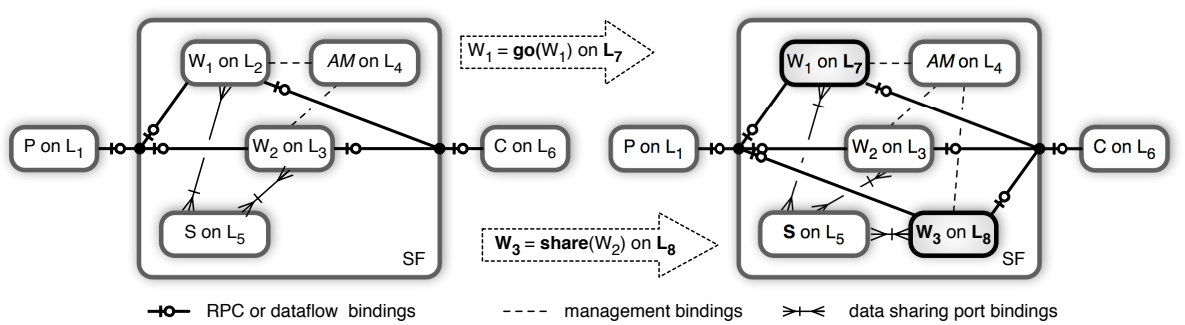

The described assembly of components (left) is paradigmatic of many producerfilter-consumer applications, where the producer $(P)$ generates a stream of data and the filter is parallel component (SF) exhibiting a shared state among its inner components (e.g. a database). The original assembly (left) can be dynamically adapted (right) by way of two adaptation operations to react to run-time events, such as a request of increasing the throughput. The go operation moves $\mathrm{W}_{1}$ from $\mathrm{L}_{2}$ to $\mathrm{L}_{7}$ (as an example to move a component onto a more powerful machine); the share operation that replicates $\mathrm{W}_{2}$ and place it in the new location $\mathrm{L}_{8}$ (to increase the parallelism degree). Both operations preserve the external state of the migrated/replicated component, which is realised by way of a storage component) attached via a data sharing interface [3].

Example 2.1 illustrates how the management can be described from a global viewpoint. Indeed, the system is described by in a rather detailed way, e.g., components are explicitly enumerated along with their connections. Even if this global viewpoint is useful (and sometime unavoidable) when designing distributed systems, it falls short in describing what single components are supposed to do when a reconfiguration is required. In other terms, it is hard to tell what the local behaviour of each component should be in order to obtain the reconfiguration described by the global view.

Also, it is worth remarking that, though the diagram clearly describes the changes triggered by $A M$ in this scenario, the lack of a formal semantics leaves some ambiguities. For example, it is not clear if the reconfiguration should take place if, and only if, the system is configured as on the lhs or this is rather a

\footnotetext{
${ }^{1}$ This component is a composite component, and in particular it is an instance of a behavioural skeleton [1].
} 
"template" configuration (e.g., should the system reconfigure itself also when $W_{2}$ is connected to $W_{1}$ rather than to $D$ ? What if $W_{2}$ was not present?). Of course, such ambiguous situations can be avoided when a formal semantics is adopted.

\section{A Walk through SHR}

Synchronised Hyperedge Replacement (SHR) can be thought of as a rule-based framework for modelling (various aspects of) of distributed computing [7] modelled as hypergraphs, a generalisation of graphs roughly representing (sets of) relations among nodes. While graphs represent (sets of) binary relations (labelled arcs connect exactly two nodes), labelled hyperedges (hereafter, edges) can connect any number of nodes. We give an informal albeit precise description of hypergraphs and SHR through a suitable graphical notation. The interested reader is referred to $[7,11]$ and references therein for the technical details.

Example 3.1 In our graphical notation, a hypergraph is depicted as

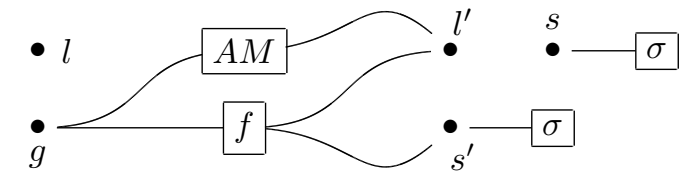

Edges (labelled by $f, A M$ and $\sigma$ ) are connected to nodes ( $g, l, l^{\prime}, s$ and $\left.s^{\prime}\right)$. Specifically, AM connects $g$ and $l^{\prime}, f$ connects $g, l^{\prime}$ and $s^{\prime}$ while two $\sigma$-labelled edges are attached to $s$ and $s^{\prime}$. Notice that nodes can be isolated (e.g., l).

Hyperedges represent (distributed) components that interact through ports represented by nodes. Connections between edges and nodes, called tentacles, allow components sharing ports to interact (e.g., in Example 3.1, $f$ and $A M$ can interact on $g$ and on $l^{\prime}$ ).

Example 3.2 The hypergraph in Example 3.1 represents (part of) a system where a manager $A M$ and a component $f$ are located at $l^{\prime}$ and can interact on port $g$. The component $f$ has access to the store at $s^{\prime}$ (e.g. by way of a data port [3]). In the system are also present another location $l$ and store $s$.

As in string grammars, SHR rewriting is driven by productions. In fact, strings can be rewritten according to a set of productions, i.e. rules of the form $\alpha \rightarrow \beta$, where $\alpha$ and $\beta$ are strings (over fixed alphabets of terminal and non-terminal symbols). Similarly, in SHR hypergraph rewritings are specified by productions of the form $L \rightarrow R$, where the lhs $L$ is a hyperedge, the rhs $R$ is a hypergraphs and states that occurrences of $L$ can be replaced with $R$. Intuitively, edges correspond to non-terminals and can be replaced with a hypergraph according to their productions. In SHR, hypergraphs are rewritten by synchronising productions, namely edge replacement is synchronised: to apply the productions of edges sharing nodes, some conditions must be fulfilled. More precisely, an SHR production can be represented as follows: 

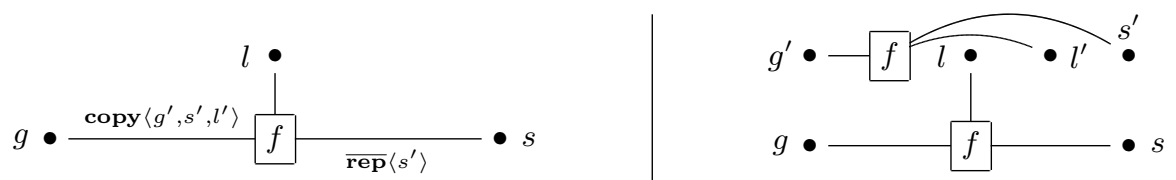

where on the lhs is a decorated edge and on the rhs a hypergraph. The production above should be read as a rewriting rule specifying that edge $f$ on the lhs can be replaced with the hypergraph on the rhs provided that the conditions on the tentacles are fulfilled. More precisely, copy and $\overline{\mathbf{r e p}}$ must be satisfied on node $g$ and $s$, respectively while $f$ is $i d l e$ on node $l$, namely it does not pose any condition on $l$. According to our interpretation, this amounts to say that when component $f$ is said to replicate with copy by its $A M$ (condition copy on node $g$ ), it tells its store to duplicate itself (condition rep on node $s$ ). When such conditions are fulfilled, edge $f$ is replaced with the hypergraph on the rhs which yield two instances of $f$ one of which connected to the communicated nodes as prescribed by the rhs of the production. Indeed, $f$ exposes three nodes on condition copy and one on rep; these represent nodes that are communicated (i.e., $g$ and $l$ are node communication accounts for mobility as edges can dynamically detach their tentacles from nodes and connect them elsewhere.

SHR has a declarative flavour because programmers specify synchronization conditions of components independently from each other. Once the system is built (by opportunely connecting its components) it will evolve according to the possible synchronizations of the edges. Global transitions are obtained by parallel application of productions with "compatible" conditions where compatibility depends on the chosen synchronisation policy ${ }^{2}$.

\section{Productions for Non-functional Interfaces}

SHR can adequately formalise the non-functional interface mechanisms informally described in Sec. 2. Three conceptually distinct interfaces can be considered: $i$ ) interfaces between components and $A M$ (for management bindings), $i i$ ) interfaces toward the external state (for data sharing bindings), and iii) interfaces for communicating with other components (for RPC/dataflow bindings). Since interfaces $i$ ii are application dependent, we focus on the coordinationrelated interfaces $i$ and $i$.

A main advantage of our approach is that all aspects of non-functional interfaces are captured in a uniform framework based on SHR. Indeed,

- components are abstracted as edges connected to form a hypergraph;

- the coordination interface of each component is separately declared and is not mingled with its computational activity;

- being SHR a local rewriting mechanism, it is possible to specify confined re-configuration of systems triggered by local conditions;

${ }^{2} \mathrm{SHR}$ is parametric with respect to the synchronisation mechanism adopted and can even encompass several synchronisation mechanisms $[7,11]$. 
Migration The migration of a component $f$ is triggered when its $A M$ raises a signal go with the new location on node $g$. The synchronisation of $f$ on the go signal is given by following production:

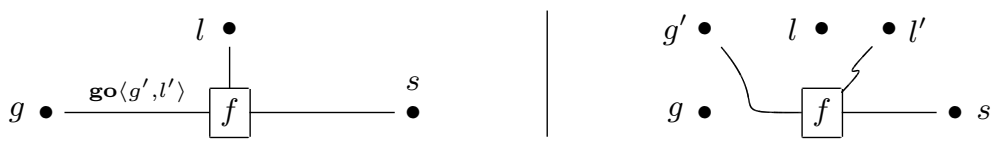

specifying that $f$ running at $l$ accepts to migrate to $l^{\prime}$ (lhs); the "location" tentacle of $f$ is disconnected from $l$ and attached to $l^{\prime}$ (rhs). Notice that $f$ maintains the connection to the previous state $s$ and $l$ is still present. The tentacle connected to $g$ on the lhs is connected to $g^{\prime}$ on the rhs; however, it might well be that $g^{\prime}=g(f$ is still connected to the original $A M)$ or $g \neq g^{\prime}(f$ changes manager). Similarly, start moves the component to a new location $l^{\prime}$. However, a new external state $\sigma$ is created together with its attaching node:

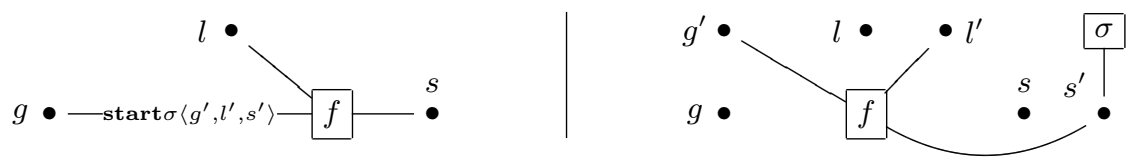

Replication Unlike migration, replication of $f$ preserves its location:
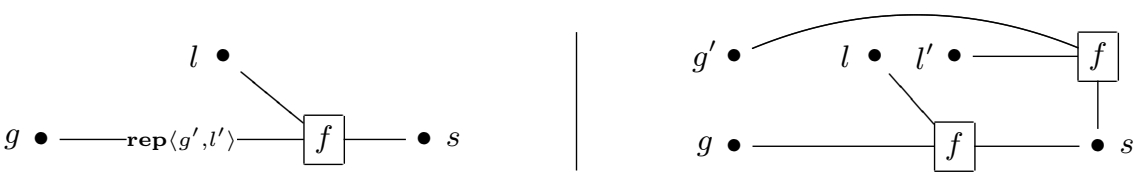

the effect of the above production is to add a new instance of $f$ at $l^{\prime}$ with $A M$ connected to $g^{\prime}$; of course, $l=l^{\prime}$ and $g=g^{\prime}$ are possible. The newly generated instance shares external state with the original one.

Replication can also activate the new instance with a different state:
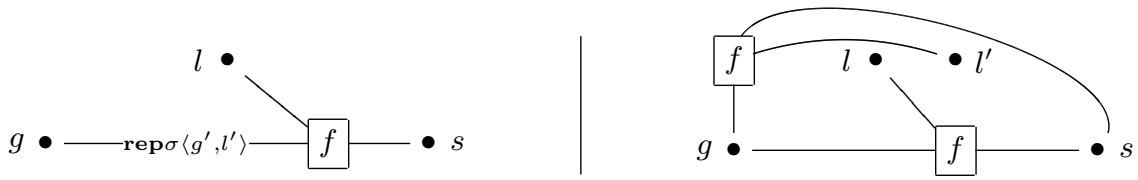

The production above creates a fresh replica of $f$ at $l^{\prime}$ and assigns to it the manager at $g^{\prime}$; notice that the two instances of $f$ share the state $s$.

Replication can also trigger a new instance of $f$ that acts on a copy of the state original state as described in the production of page 5 where $f$ must notify to its state to duplicate itself and connect the new copy on $s^{\prime}$. Hence, the state connected to $s$ duplicate itself on the node $s^{\prime}$ when the action complementary to $\overline{\mathbf{r e p}}$ is received, as stated below.

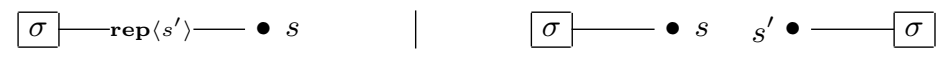


Component killing Components are killed using the following production:
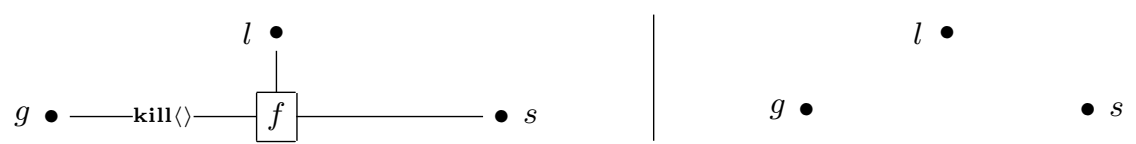

stating that $f$ disappears when its corresponding $A M$ sends a kill signal.

\section{Synchronizing productions}

The operational semantics of SHR is illustrated through an example that highlights the following steps:

1. individuate the adjacent tentacles labeled by compatible conditions;

2. determine the synchronizing productions and replace the (instances of) edges on their lhs with the hypergraphs on their rhs;

3 . fuse the nodes that are equated by the synchronizations.

Let us apply the previous steps to show how migration works in a situation represented by the following hypergraph

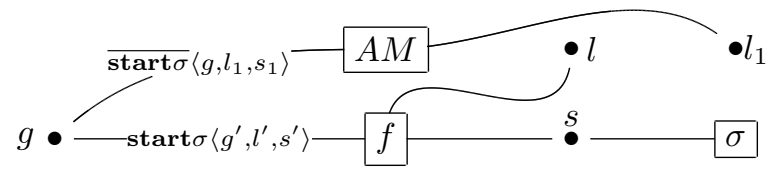

where component $f$ is running at $l$ and shares $g$ with a manager $A M$ located at $l_{1}$. For brevity, tentacles are decorated with the conditions triggering the rewriting (step 1). Indeed, the tentacles of $A M$ and of $f$ incident on node $g$ yield compatible output and input conditions respectively so that $A M$ orders $f$ to migrate to $l_{1}$ and to use the store at $s_{1}$ while staying connected to $g$.

Productions synchronisation consists in replacing the occurrences of the edges on the lhs with the hypergraphs specified in the rhs of the productions and applying the node fusions obtained by the node communicated. For instance, in the previous example the synchronising productions are the start $\sigma$ production of $f$ given in Sec. 4 and the production of $A M$ whose lhs and rhs consist of $A M$ connected to $g$ and $l_{1}$ (step 2). Hence, after the synchronization, the node fusions $g^{\prime}=g, l^{\prime}=l_{1}$ and $s^{\prime}=s_{1}$ are applied (step 3), so that the hypergraph is rewritten as

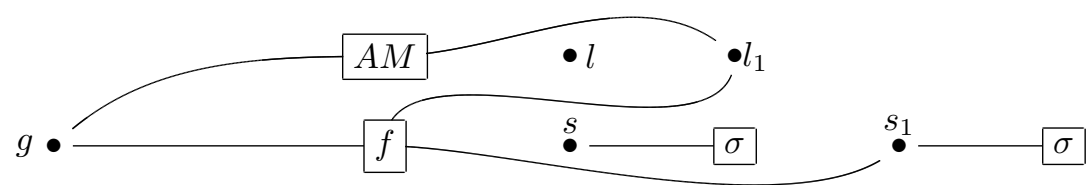

Let us remark that $l, \sigma$ and $s$ remains in the final hypergraph. In fact they should not be removed because other edges can be allocated on $l$ or access $\sigma$.

The intuitive description of SHR given in this section suggests the following design style and execution style: 
- assign an edge to each component and specify their productions;

- represent the system as a hypergraph;

- decorate the tentacles with the synchronisation conditions;

- synchronize the productions until possible.

It is worth remarking that, unlike other semantical frameworks (e.g., process calculi), in SHR synchronisation conditions may require more than two (productions of) components to be synchronised. This actually depends on the synchronisation policy at hand. For instance, in the migration rewriting described in this section, it is possible to use broadcast interactions on the node $g$ so that all the components connected on $g$ will move at $l^{\prime}$ when the productions are synchronised.

\section{Conclusions}

The SHR is one of the modelling and theoretical frameworks of the SEnsoria project [14]. SHR has been exploited in [6] for managing application level service level agreement (SLA) in a distributed environment; in [7] several variants of SHR have been described in a uniform context showing how this formalism can suitably tackle many programming and modelling facets of arising in service oriented computing. In this paper we have shown how SHR can be suitably used to formalise both adaption operations and the evolution of componentbased autonomic applications.

In an autonomic component model, as GCM, the $A M$ relies on adaptation operation to realise its management policy (during the execution phase of the autonomic cycle). In general, a proper formalisation of adaptation operations (possibly involving the $A M$ itself), may help

- to develop correct and effective management policies providing the developer with a precise description of the effect (semantics) adapation operations;

- to formally prove the proprierties of a single adaptations or sequences of them;

- to formally prove local or global invariants characterising the evolution of assemblies of autonomic components;

- to guide developers of the component model itself to establish the effectiveness of provided adaptation operations (e.g. the completeness of the set of operations with respect to a given planned evolution of the assembly).

This work covers the first of these items, thus represents a first step in this roadmap, whereas successive items can be enumerated in the future work. The presented adaptation operations are currently implemented in the reference implementation of GCM (developed within the GridCOMP STREP project [8]); their effectiveness in managing the QoS of grid applications is reported in [1]. 


\section{Acknowledgments}

The final publication is available at springerlink.com:

M. Aldinucci and E. Tuosto. Towards a formal semantics for autonomic components. In T. Priol and M. Vanneschi, editors, From Grids To Service and Pervasive Computing (Proc. of the CoreGRID Symposium 2008), CoreGRID, pages 31-45, Las Palmas, Spain, Aug. 2008. Springer. DOI: 10.1007/978-0387-09455-7_3

\section{References}

[1] M. Aldinucci, S. Campa, M. Danelutto, M. Vanneschi, P. Dazzi, D. Laforenza, N. Tonellotto, and P. Kilpatrick. Behavioural skeletons in GCM: autonomic management of grid components. In D. E. Baz, J. Bourgeois, and F. Spies, editors, Proc. of Intl. Euromicro PDP 2008: Parallel Distributed and network-based Processing, pages 54-63, Toulouse, France, Feb. 2008. IEEE.

[2] M. Aldinucci and M. Danelutto. Algorithmic skeletons meeting grids. Parallel Computing, 32(7):449-462, 2006.

[3] G. Antoniu, H. Bouziane, L. Breuil, M. Jan, and C. Pérez. Enabling transparent data sharing in component models. In 6th IEEE Intl. Symposium on Cluster Computing and the Grid (CCGRID), pages 430-433, Singapore, May 2006.

[4] CoreGRID NoE deliverable series, Institute on Programming Model. Deliverable D.PM.04 - Basic Features of the Grid Component Model (assessed), Feb. 2007.

[5] P.-C. David and T. Ledoux. An aspect-oriented approach for developing self-adaptive fractal components. In W. Löwe and M. Südholt, editors, Proc. of the 5th Intl Symposium Software on Composition (SC 2006), volume 4089 of $L N C S$, pages 82-97, Vienna, Austria, Mar. 2006. Springer.

[6] R. De Nicola, G. Ferrari, U. Montanari, R. Pugliese, and E. Tuosto. A formal basis for reasoning on programmable Qos. In Intl. Symposium on Verification - Theory and Practice - Honoring Z. Manna's 64th Birthday, volume 2772 of LNCS. Springer, June 2003.

[7] G. Ferrari, D. Hirsch, I. Lanese, U. Montanari, and E. Tuosto. Synchronised hyperedge replacement as a model for service oriented computing. In F. de Boer, M. Bonsangue, S. Graf, and W. de Roever, editors, Formal Methods for Components and Objects: 4th Intl. Symposium, FMCO, volume 4111 of LNCS, Amsterdam, The Netherlands, Nov. 2006. Springer. Revised Lectures. 
[8] GridCOMP. GridCOMP web page, 2007. http://gridcomp.ercim.org.

[9] K. Kennedy, M. Mazina, J. Mellor-Crummey, K. Cooper, L. Torczon, F. Berman, A. Chien, H. Dail, O. Sievert, D. Angulo, I. Foster, D. Gannon, L. Johnsson, C. Kesselman, R. Aydt, D. Reed, J. Dongarra, S. Vadhiyar, and R. Wolski. Toward a framework for preparing and executing adaptive Grid programs. In Proc. of NSF Next Generation Systems Program Workshop (IPDPS 2002), 2002.

[10] J. O. Kephart and D. M. Chess. The vision of autonomic computing. IEEE Computer, 36(1):41-50, 2003.

[11] I. Lanese and E. Tuosto. Synchronized Hyperedge Replacement for Heterogeneous Systems. In J. Jacquet and G. Picco, editors, International Conference on Coordination Models and Languages, volume 3454 of LNCS, pages 220 - 235. Springer, April 2005.

[12] ObjectWeb Consortium. The Fractal Component Model, Technical Specifcation, 2003.

[13] M. Parashar, H. Liu, Z. Li, V. Matossian, C. Schmidt, G. Zhang, and S. Hariri. AutoMate: Enabling autonomic applications on the Grid. Cluster Computing, 9(2):161-174, 2006.

[14] Sensoria Project. Software Engineering for Service-Oriented Overlay Computers. Public Web Site. http://sensoria.fast.de/.

[15] M. Vanneschi. The programming model of ASSIST, an environment for parallel and distributed portable applications. Parallel Computing, 28(12):1709-1732, Dec. 2002. 